\title{
Generalized Chernoff Information for Mismatched Bayesian Detection and Its Application to Energy Detection
}

Yuni Lee, Student Member, IEEE, and Youngchul Sung, Senior Member, IEEE

\begin{abstract}
In this letter, the performance of mismatched likelihood ratio detectors for binary Bayesian hypothesis testing problems is considered. Based on large deviation theory, a method for achieving the maximum Bayesian error exponent for a mismatched likelihood ratio detector is presented. It is shown that the maximum Bayesian error exponent is given by generalized Chernoff information, which is an extension of the Chernoff information to the case of two mismatched distributions and has similar properties to those of the original Chernoff information. As an application example, energy detection under the Gauss-Markov signal model, is considered. It is shown that the generalized Chernoff information of energy detection, which is achieved by optimally choosing the detection threshold, is close to the original Chernoff information for the considered signal model, and thus, the performance of suboptimal energy detection can be improved significantly simply by choosing the detection threshold judiciously.
\end{abstract}

Index Terms - Chernoff information, energy detection, error exponent, large deviation theory, mismatched likelihood ratio detection.

\section{INTRODUCTION}

$\mathbf{F}$ OR binary Bayesian detection problems, the optimal decision rule that minimizes the Bayesian error probability is the log-likelihood ratio test (LLRT). However, due to inaccurate distribution information or complexity reasons, the exact LLRT cannot be adopted in many applications. In certain cases, the adopted suboptimal detector can be represented as a mismatched (log-)likelihood ratio detector, i.e., an LLRT based on null and alternative distributions that are not the same as true underlying distributions [2]. Several works have been done in this area under the framework of minimax robust detection, which focuses on finding an optimal detector for the least favorable condition within a given class of underlying distributions [3]-[7]. However, not much attention was given to analyzing the performance of mismatched detectors. In this letter, we

Manuscript received May 23, 2012; accepted August 10, 2012. Date of publication August 27, 2012; date of current version September 13, 2012. This work was supported in part by Basic Science Research Program through the National Research Foundation of Korea (NRF) funded by the Ministry of Education, Science and Technology (2010-0021269) and by the Basic Science Research Program through the S\&T Reading Primary Research funded by KAIST (N10110050). Parts of this work were presented in [1], but the focus and contents of this letter are quite different from [1]. The associate editor coordinating the review of this manuscript and approving it for publication was Dr. Peng Qiu.

The authors are with the Department of Electrical Engineering, KAIST, Daejeon 305-701, South Korea (e-mail: yunilee@kaist.ac.kr; ysung@ee.kaist.ac. $\mathrm{kr})$.

Digital Object Identifier 10.1109/LSP.2012.2215585 focus on the mismatched detection problem itself, analyze the performance of mismatched likelihood ratio detectors, and propose a new asymptotic performance measure, referred to as generalized Chernoff information, for mismatched likelihood ratio detectors, based on large deviations theory. Whereas the Bayesian error exponent is given by the Chernoff information between the true null and alternative distributions [8], the maximum Bayesian error exponent for a mismatched likelihood ratio detector is given by the proposed generalized Chernoff information, which is obtained by a certain equalizer rule applied to the Fenchel-Legendre transforms of mismatched log-generating functions and attained by optimally choosing the detection threshold. We show that the generalized Chernoff information is nonnegative and symmetric between the null and alternative distributions and reduces to the original Chernoff information if the detection statistic is the true log-likelihood ratio. For an application example, we consider the energy detection of a zero-mean stationary Gaussian signal in white Gaussian noise. In this case, the energy detector is a mismatched likelihood ratio detector when the signal to be detected is not independent and identically distributed (i.i.d.). By applying the proposed method, we compute the generalized Chernoff information for the energy detection under the Gauss-Markov signal correlation model. Under the considered Gauss-Markov signal model, numerical results show that the generalized Chernoff information is close to the Chernoff information. Thus, there is only a small loss in the error exponent simply by properly choosing the detection threshold even if the signal correlation is neglected.

The remainder of the letter is organized as follows. In Section II, we describe the data model and formulate the problem. Generalized Chernoff information for mismatched detectors is defined and analyzed in Section III. Then, it is applied to the energy detection for stationary Gaussian signals under the Gauss-Markov signal model in Section IV, followed by conclusion in Section V.

\section{Data Model and Problem Statement}

We consider the following binary hypothesis problem:

$$
\begin{array}{ll}
\mathcal{H}_{0}: & \mathbf{x}_{n} \sim p_{0, n} \\
\mathcal{H}_{1}: & \mathbf{x}_{n} \sim p_{1, n},
\end{array}
$$


where $\mathbf{x}_{n}:=\left[x_{1}, x_{2}, \ldots, x_{n}\right]$ is the random observation vector of size $n$. Under the Bayesian criterion, the optimal detector for the problem (1) is the (normalized) LLRT:

$$
T_{n}:=\frac{1}{n} \log \left(\frac{p_{1, n}(\mathbf{x})}{p_{0, n}(\mathbf{x})}\right)_{\mathcal{H}_{0}}{ }^{\mathcal{H}_{1}} \frac{1}{n} \log \frac{\pi_{0}}{\pi_{1}}=: \tau^{\star},
$$

where $\pi_{j}, j=0,1$, is the prior probability for the hypothesis $\mathcal{H}_{j}$. The maximum error exponent (or exponential decaying rate) of the Bayesian error probability $P_{e}$ for the optimal detector is given by the Chernoff information [8]:

$$
\begin{aligned}
- & \lim _{n \rightarrow \infty} \frac{1}{n} \log P_{e} \\
& =-\inf _{0 \leq u \leq 1} \lim _{n \rightarrow \infty} \frac{1}{n} \log \int p_{0, n}^{u}(\mathbf{x}) p_{1, n}^{1-u}(\mathbf{x}) d \mathbf{x} \\
& =: C\left(p_{0} ; p_{1}\right),
\end{aligned}
$$

where $P_{e}=\pi_{0} P_{F}+\pi_{1} P_{M}$ and $P_{F}$ and $P_{M}$ are the false alarm and miss detection probabilities, respectively. When there is uncertainty in the underlying null and/or alternative distributions or when the complexity of the optimal detector is too high, one may employ a suboptimal detector, and in many cases the suboptimal detector is represented by a mismatched likelihood ratio detector [2]:

$$
\tilde{T}_{n}:=\frac{1}{n} \log \left(\frac{\tilde{p}_{1, n}(\mathbf{x})}{\tilde{p}_{0, n}(\mathbf{x})}\right) \underset{\mathcal{H}_{0}}{\geq}{ }_{\mathcal{H}_{1}} \tau
$$

which discriminates the two incorrect hypotheses

$$
\begin{array}{ll}
\tilde{\mathcal{H}}_{0}: & \mathbf{x}_{n} \sim \tilde{p}_{0, n} \\
\tilde{\mathcal{H}}_{1}: & \mathbf{x}_{n} \sim \tilde{p}_{1, n},
\end{array}
$$

In the following section, we shall derive the maximum error exponent for this mismatched detector and evaluate the asymptotic performance of this mismatched detector by using the large deviation principle.

\section{Generalized Chernoff Information}

To obtain the maximum Bayesian error exponent for the mismatched detector, we first need the log-generating functions under the null and alternative hypotheses:

$$
\begin{aligned}
\tilde{\Lambda}_{0}(u) & =\lim _{n \rightarrow \infty} \frac{1}{n} \log \mathbb{E}\left\{\exp \left(n u \tilde{T}_{n}\right) \mid \mathcal{H}_{0}\right\} \\
& =\lim _{n \rightarrow \infty} \frac{1}{n} \log \int\left[\tilde{p}_{1, n}(\mathbf{x})\right]^{u}\left[\tilde{p}_{0, n}(\mathbf{x})\right]^{-u} p_{0, n}(\mathbf{x}) d \mathbf{x} \\
\tilde{\Lambda}_{1}(u), & =\lim _{n \rightarrow \infty} \frac{1}{n} \log \mathbb{E}\left\{\exp \left(n u \tilde{T}_{n}\right) \mid \mathcal{H}_{1}\right\} \\
& =\lim _{n \rightarrow \infty} \frac{1}{n} \log \int\left[\tilde{p}_{1, n}(\mathbf{x})\right]^{u}\left[\tilde{p}_{0, n}(\mathbf{x})\right]^{-u} p_{1, n}(\mathbf{x}) d \mathbf{x} .
\end{aligned}
$$

One can easily show by applying Hölder's inequality that $\tilde{\Lambda}_{0}$ and $\tilde{\Lambda}_{1}$ are convex. Note that $\tilde{\Lambda}_{0}(0)=\tilde{\Lambda}_{1}(0)=0$. For the mismatched likelihood ratio detector (4), the error exponents for the false alarm and miss detection probabilities are given by the Gärtner-Ellis theorem [8]. That is, the error exponents of the false alarm and miss detection probabilities are obtained from the Fenchel-Legendre transforms of the corresponding log-generating functions under certain mild conditions: ${ }^{1}$ :

$$
\begin{gathered}
E_{F}(\tau) \triangleq-\lim _{n \rightarrow \infty} \frac{1}{n} \log \left(\operatorname{Pr}\left\{\tilde{T}_{n} \geq \tau \mid \mathcal{H}_{0}\right\}\right)=\inf _{t \geq \tau} \tilde{\Lambda}_{0}^{*}(t), \\
E_{M}(\tau) \triangleq-\lim _{n \rightarrow \infty} \frac{1}{n} \log \left(\operatorname{Pr}\left\{\tilde{T}_{n}<\tau \mid \mathcal{H}_{1}\right\}\right)=\inf _{t<\tau} \tilde{\Lambda}_{1}^{*}(t),
\end{gathered}
$$

where $\tilde{\Lambda}_{i}^{*}(t), i=0,1$ is the Fenchel-Legendre transform of $\tilde{\Lambda}_{i}$, i.e.,

$$
\tilde{\Lambda}_{i}^{*}(t)=\sup _{u}\left\{t u-\tilde{\Lambda}_{i}(u)\right\}
$$

Since $\tilde{\Lambda}_{i}(u)$ is convex, the supremum of $\left\{t u-\tilde{\Lambda}_{i}(u)\right\}$ occurs when $u$ satisfies $(d) /(d u) \tilde{\Lambda}_{i}(u)=t$. Thus, an intuitive explanation for the Fenchel-Legendre transform is the negative value of $y$-intercept of the tangent line of $\tilde{\Lambda}_{i}(u)$ that has slope $t$.

Lemma 1: Suppose that $\tilde{\Lambda}_{0}(u)$ is differentiable on the interior of its domain. Then, $\tilde{\Lambda}_{0}^{*}(t)$ is a non-decreasing function of $t$ for $t \geq(d) /(d u) \tilde{\Lambda}_{0}(0)$.

Proof: $(d) /(d u) \tilde{\Lambda}_{0}(u)$ is an increasing function since $\tilde{\Lambda}_{0}$ is convex. Therefore, for $t \geq(d) /(d u) \tilde{\Lambda}_{0}(0)$, the supremum of $\left\{t u-\tilde{\Lambda}_{0}(u)\right\}$ occurs at $u>0$, i.e.,

$$
\tilde{\Lambda}_{0}^{*}(t)=\sup _{u}\left\{t u-\tilde{\Lambda}_{0}(u)\right\}=\sup _{u \geq 0}\left\{t u-\tilde{\Lambda}_{0}(u)\right\} .
$$

Let $t^{\prime} \geq t\left(\geq(d) /(d u) \tilde{\Lambda}_{0}(0)\right)$. Then, for any $u \geq 0$ we have

$$
t u-\tilde{\Lambda}_{0}(u) \leq t^{\prime} u-\tilde{\Lambda}_{0}(u) \leq \sup _{u \geq 0}\left\{t^{\prime} u-\tilde{\Lambda}_{0}(u)\right\} .
$$

Therefore, we have $\tilde{\Lambda}_{0}^{*}(t)=\sup _{u \geq 0}\left\{t u-\tilde{\Lambda}_{0}(u)\right\} \leq$ $\tilde{\Lambda}_{0}^{*}\left(t^{\prime}\right)$.

Using similar arguments, we also have the following lemma.

Lemma 2: Suppose that $\tilde{\Lambda}_{1}$ is differentiable on the interior of its domain. Then, $\tilde{\Lambda}_{1}^{*}(t)$ is a non-increasing function of $t$ for $t \leq(d) /(d u) \tilde{\Lambda}_{1}(0)$.

Thus, by Lemmas 1 and 2, we have $E_{F}(\tau)=\inf _{t \geq \tau} \tilde{\Lambda}_{0}^{*}(t)=$ $\tilde{\Lambda}_{0}^{*}(\tau)$ and $E_{M}(\tau)=\inf _{t<\tau} \tilde{\Lambda}_{1}^{*}(t)=\tilde{\Lambda}_{1}^{*}(\tau)$. Since the Bayesian error probability is a weighted sum of the false alarm and miss detection probabilities, i.e., $P_{e}=\pi_{0} P_{F}+\pi_{1} P_{M}$, it is asymptotically dominated by the term that has the lower decaying rate. Thus, the Bayesian error exponent $E(\tau)$ is given by

$$
E(\tau)=\min \left\{E_{F}(\tau), E_{M}(\tau)\right\}=\min \left\{\tilde{\Lambda}_{0}^{*}(\tau), \tilde{\Lambda}_{1}^{*}(\tau)\right\} .
$$

Definition 1: (Generalized Chernoff information) The generalized Chernoff information $C\left(p_{0} \rightarrow \tilde{p}_{0} ; p_{1} \rightarrow \tilde{p}_{1}\right)$ for the mismatched distributions $\tilde{p}_{0, n}$ and $\tilde{p}_{1, n}$ is defined as the maximum

\footnotetext{
${ }^{1}$ We assume that for the considered problem, the conditions are satisfied.
} 
Bayesian error exponent of the mismatched likelihood ratio detector (4), i.e.,

$$
\begin{aligned}
C\left(p_{0} \rightarrow \tilde{p}_{0} ; p_{1} \rightarrow \tilde{p}_{1}\right) & :=\max _{\tau} E(\tau) \\
& =\max _{\tau} \min \left\{\tilde{\Lambda}_{0}^{*}(\tau), \tilde{\Lambda}_{1}^{*}(\tau)\right\} .
\end{aligned}
$$

The following theorem states how to compute the generalized Chernoff information.

Theorem 1: The generalized Chernoff information for the mismatched distributions $\tilde{p}_{0, n}$ and $\tilde{p}_{1, n}$ (or equivalently maximum Bayesian error exponent for the mismatched likelihood ratio detector (4)) is attained by the optimal threshold $\tau_{\text {opt }}$ satisfying the following equalizer rule:

$$
\tilde{\Lambda}_{0}^{*}\left(\tau_{\mathrm{opt}}\right)=\tilde{\Lambda}_{1}^{*}\left(\tau_{\mathrm{opt}}\right)
$$

and the optimal threshold is obtained by simultaneously solving the following two equations of $u_{0}$ and $u_{1}$ :

$$
\begin{aligned}
& \frac{d}{d u} \tilde{\Lambda}_{0}\left(u_{0}\right)=\frac{d}{d u} \tilde{\Lambda}_{1}\left(u_{1}\right) \\
& {\left[\tilde{\Lambda}_{0}\left(u_{0}\right)+\left(u-u_{0}\right) \frac{d}{d u} \tilde{\Lambda}_{0}\left(u_{0}\right)\right]_{u=0}} \\
& \quad=\left[\tilde{\Lambda}_{1}\left(u_{1}\right)+\left(u-u_{1}\right) \frac{d}{d u} \tilde{\Lambda}_{1}\left(u_{1}\right)\right]_{u=0}
\end{aligned}
$$

as $\tau_{\text {opt }}=(d) /(d u) \tilde{\Lambda}_{0}\left(u_{0}\right)=(d) /(d u) \tilde{\Lambda}_{1}\left(u_{1}\right)$.

Proof: The maximum $E(\tau)$ is attained when $\min \left\{\tilde{\Lambda}_{0}^{*}(\tau), \tilde{\Lambda}_{1}^{*}(\tau)\right\}$ is maximized. Since $\tilde{\Lambda}_{0}^{*}(\tau)$ is a non-decreasing function of $\tau$, and $\tilde{\Lambda}_{1}^{*}(\tau)$ is a non-increasing function of $\tau$ by Lemmas 1 and 2, the minimum of the two is maximized when they are the same. Therefore, we have (15). By the properties of the Fenchel-Legendre transform, $\tau$ is the slope of the tangent line to $\tilde{\Lambda}_{i}(u)$, and $\tilde{\Lambda}_{i}^{*}(\tau)$ is the $y$-intercept of the tangent line. Thus, for (15), two tangent lines (one for $\tilde{\Lambda}_{0}(u)$ and the other for $\tilde{\Lambda}_{1}(u)$ ) should have the same slope, yielding (16), and the same $y$-intercept, yielding (17).

Fig. 1 illustrates the situation. In the case of the exact LLRT, the optimal threshold is zero, $\tau^{\star}=0$, in the asymptotic regime, regardless of the prior probabilities. Furthermore, in this case, we have $\Lambda_{1}(u)=\Lambda_{0}(u+1)$ for the exact distributions. That is, $\Lambda_{1}(u)$ is a shifted version of $\Lambda_{0}(u)$. Thus, the maximum Bayesian error exponent is attained by $\tau^{\star}=0$. However, in the mismatched case, the zero threshold $\tau^{\star}$, which is optimal for the exact LLRT, is always suboptimal for the mismatched detection. Now, we provide a few properties of the generalized Chernoff information. First, if we use exact distributions, i.e., $\tilde{p}_{i}=p_{i}$ for $i=0,1$, then $\tau_{\text {opt }}$ becomes $\tau^{\star}=0$ and $C\left(p_{0} \rightarrow \tilde{p}_{0} ; p_{1} \rightarrow \tilde{p}_{1}\right)$ is equivalent to the original Chernoff information $C\left(p_{0} ; p_{1}\right)$ for exact distribution. This is because, in the case of exact distributions, (6) and (7) become exact log-generating functions. The nonnegativity of the generalized Chernoff information is easily shown since $\tilde{\Lambda}_{i}^{*}(t)=\sup _{u}\left\{t u-\tilde{\Lambda}_{i}(u)\right\} \geq 0$. This is because at $u=0, t \cdot 0-\tilde{\Lambda}_{i}(0)=0$ and $\tilde{\Lambda}_{i}^{*}(t)$ has the monotone property by Lemmas 1 and 2 . Finally, the generalized Chernoff information is symmetric with respect to $p_{0, n}$ and $p_{1, n}$, as the original

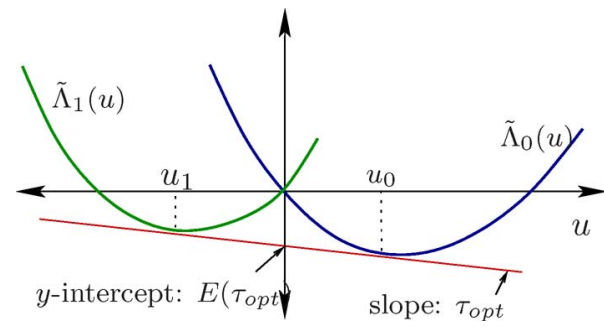

Fig. 1. Graphical illustration for $\tau_{\mathrm{opt}}$ and $E_{\mathrm{opt}}$.

Chernoff information, provided that the mismatched distributions are also switched accordingly. To see this, let $\Gamma_{i}, i=0,1$ be the switched log-generating functions:

$$
\begin{aligned}
\Gamma_{0}(u) & =\lim _{n \rightarrow \infty} \frac{1}{n} \log \int\left[\tilde{p}_{0, n}(\mathbf{x})\right]^{u}\left[\tilde{p}_{1, n}(\mathbf{x})\right]^{-u} p_{1, n}(\mathbf{x}) d \mathbf{x} \\
& =\tilde{\Lambda}_{1}(-u) \\
\Gamma_{1}(u) & =\lim _{n \rightarrow \infty} \frac{1}{n} \log \int\left[\tilde{p}_{0, n}(\mathbf{x})\right]^{u}\left[\tilde{p}_{1, n}(\mathbf{x})\right]^{-u} p_{0, n}(\mathbf{x}) d \mathbf{x} \\
& =\tilde{\Lambda}_{0}(-u) .
\end{aligned}
$$

The corresponding Fenchel-Legendre transform can be expressed in terms of $\tilde{\Lambda}_{i}^{*}$ as

$$
\begin{aligned}
\Gamma_{0}^{*}(t) & =\sup _{u}\left\{t u-\Gamma_{0}(u)\right\}=\sup _{u}\left\{t u-\tilde{\Lambda}_{1}(-u)\right\} \\
& =\sup _{u}\left\{-t u-\tilde{\Lambda}_{1}(u)\right\}=\tilde{\Lambda}_{1}^{*}(-t) .
\end{aligned}
$$

Similarly, $\Gamma_{1}^{*}(t)=\tilde{\Lambda}_{0}^{*}(-t)$. Therefore, by Lemmas 1 and 2, $\Gamma_{0}(t)$ is a non-decreasing function of $t$ and $\Gamma_{1}(t)$ is a non-increasing function of $t$ for $(d) /(d u) \tilde{\Lambda}_{0}(0) \leq t \leq$ $(d) /(d u) \tilde{\Lambda}_{1}(0)$. Therefore, the maximum error exponent (i.e., generalize Chernoff information) is achieved if $\Gamma_{0}^{*}(\tau)=\Gamma_{1}^{*}(\tau)$ or equivalently $\tilde{\Lambda}_{0}^{*}(-\tau)=\tilde{\Lambda}_{1}^{*}(-\tau)$. The optimal threshold for the switched problem is $\tau=-\tau_{\mathrm{opt}}$ and the generalized Chernoff information $C\left(p_{1} \rightarrow \tilde{p}_{1} ; p_{0} \rightarrow \tilde{p}_{0}\right)$ is given by

$$
\begin{aligned}
& C\left(p_{1} \rightarrow \tilde{p}_{1} ; p_{0} \rightarrow \tilde{p}_{0}\right) \\
& \quad=\Gamma_{0}^{*}\left(-\tau_{\mathrm{opt}}\right) \\
& \quad=\tilde{\Lambda}_{1}^{*}\left(\tau_{\mathrm{opt}}\right)=C\left(p_{0} \rightarrow \tilde{p}_{0} ; p_{1} \rightarrow \tilde{p}_{1}\right) .
\end{aligned}
$$

\section{Application: Threshold Optimization FOR ENERGY DETECTION}

In the previous section, we introduced the generalized Chernoff information as the maximum Bayesian error exponent for a mismatched Bayesian detection problem and showed that the generalized Chernoff information is achieved simply by choosing the detection threshold optimally. In this section, we provide a useful application example. Here, we consider the detection of a stationary Gaussian process in white Gaussian noise. This problem can be formulated in terms of the power spectra as follows:

$$
\begin{aligned}
\mathcal{H}_{0}: & S_{x}(\omega)=S_{0}(\omega)=\sigma^{2}, \quad-\pi \leq \omega<\pi, \\
\mathcal{H}_{1}: \quad & S_{x}(\omega)=S_{1}(\omega)=\theta^{2} S_{s}(\omega)+\sigma^{2}, \\
& -\pi \leq \omega<\pi,
\end{aligned}
$$


where $S_{s}(\omega)$ and $\sigma^{2}$ indicate the signal and noise power spectra, respectively. Here, we assume that the normalized signal spectrum $S_{s}(\omega)$ has unit power, i.e., $(1) /(2 \pi) \int_{\pi}^{\pi} S_{s}(\omega) d \omega=1$. Thus, the total signal-to-noise ratio (SNR) is given by $\left(\theta^{2}\right) /\left(\sigma^{2}\right)$. In many applications, due to the unavailability of $S_{s}(\omega)$ or the complexity of the exact LLRT, simply energy detection is used. The energy detection neglecting the signal correlation is an example of mismatched detection and can be expressed as

$$
\begin{aligned}
& \tilde{\mathcal{H}}_{0}: \quad S_{x}=S_{0}(\omega)=\sigma^{2}=: P_{0}, \quad-\pi \leq \omega<\pi, \\
& \tilde{\mathcal{H}}_{1}: \quad S_{x}=\tilde{S}_{1}(\omega)=\theta^{2}+\sigma^{2}=: P_{1}, \quad-\pi \leq \omega<\pi .
\end{aligned}
$$

By the Toeplitz distribution theorem, the log-generating functions are derived as

$$
\begin{aligned}
\tilde{\Lambda}_{0}(u)= & -\frac{1}{4 \pi} \int_{-\pi}^{\pi}\left[\log \left(\frac{u}{\tilde{S}_{1}(\omega)}+\frac{1-u}{S_{0}(\omega)}\right)\right. \\
& \left.+u \log \tilde{S}_{1}(\omega)+(1-u) \log S_{0}(\omega)\right] d \omega \\
\tilde{\Lambda}_{1}(u)= & -\frac{1}{4 \pi} \int_{-\pi}^{\pi}\left[\log \left(\frac{1}{S_{1}(\omega)}+\frac{u}{\tilde{S}_{1}(\omega)}-\frac{u}{S_{0}(\omega)}\right)\right. \\
& \left.+\log S_{1}(\omega)+u \log \tilde{S}_{1}(\omega)-u \log S_{0}(\omega)\right] d \omega .
\end{aligned}
$$

Regarding the log-generating functions for the distributions (23), we have the following property.

Lemma 3: For flat spectra $S_{0}(\omega)=P_{0}$ and $\tilde{S}_{1}(\omega)=P_{1}=$ $(1) /(2 \pi) \int_{-\pi}^{\pi} \tilde{S}_{1}(\omega) d \omega=(1) /(2 \pi) \int_{-\pi}^{\pi} S_{1}(\omega) d \omega$ over $[-\pi, \pi)$ with $P_{0} \leq P_{1}$, we have $\tilde{\Lambda}_{1}(u) \geq \tilde{\Lambda}_{0}(u+1)$ for all $u$.

Lemma 3 can be proved by using the concavity of logarithm and Jensen's inequality. Based on this result, we provide a general property of the optimal threshold $\tau_{\text {opt }}$ for the energy detection (23).

Theorem 2: For the energy detection (23), we have $\tau_{\mathrm{opt}} \leq 0$.

Proof: Suppose that $\tau>0$. Let $u_{1} \in \mathbb{R}$ be such that $u_{1}=$ $\arg \sup _{u}\left\{\tau u-\tilde{\Lambda}_{1}(u)\right\}$. Then

$$
\begin{aligned}
\tilde{\Lambda}_{1}^{*}(\tau) & =\tau u_{1}-\tilde{\Lambda}_{1}\left(u_{1}\right) \leq \tau u_{1}-\tilde{\Lambda}_{0}\left(u_{1}+1\right) \\
& <\tau\left(u_{1}+1\right)-\tilde{\Lambda}_{0}\left(u_{1}+1\right) \leq \sup _{u}\left\{\tau u-\tilde{\Lambda}_{0}(u)\right\} \\
& =\tilde{\Lambda}_{0}^{*}(\tau),
\end{aligned}
$$

where the first inequality is valid by Lemma 3 . Since $\tilde{\Lambda}_{1}^{*}(\tau)<$ $\tilde{\Lambda}_{0}^{*}(\tau)$, the equalizer rule (15) is not satisfied for $\tau>0$ and we have the claim.

Interestingly, Theorem 2 states that we should always favor the alternative hypothesis when we use energy detection neglecting the signal correlation, whatever the signal correlation is.

Fig. 2 shows the error exponents of three detectors, the exact LLRT, the proposed detector and the energy detector, for detection of the Gauss-Markov signal $s[n]$ with correlation $\mathbb{E}\{s[i] s[j]\}=a^{|i-j|}$ in white Gaussian noise. As seen in Fig. 2, a noticeable loss for the suboptimal threshold $\tau^{\star}=0$ exists

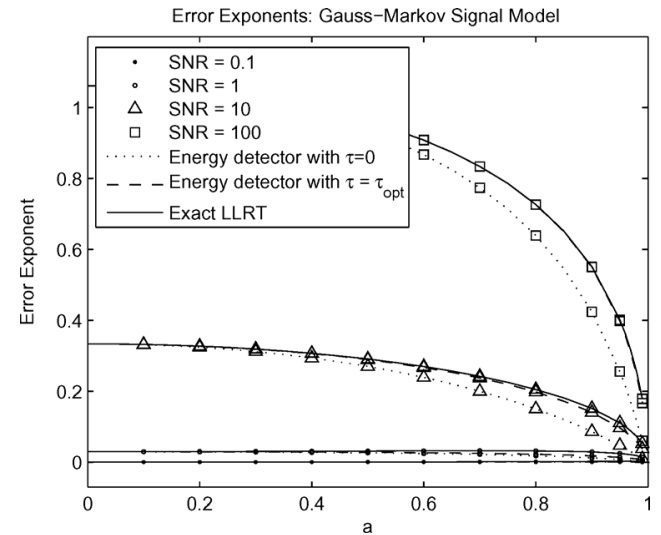

Fig. 2. Gauss-Markov signal model: Error exponent versus correlation coefficient $a$.

in the strong correlation region. However, the error exponent achieved by the optimal detection threshold is almost the same as the original Chernoff information with the exact LLRT. Although it is not clearly seen in Fig. 2, the performance loss of neglecting signal correlation is severer at low SNR [9].

\section{CONCLUSION}

In this letter, we have investigated the performance of mismatched likelihood ratio detectors for binary Bayesian hypothesis testing problems. Using large deviation theory, we have derived the maximum Bayesian error exponent for a mismatched detector and have shown that the maximum Bayesian error exponent is given by the generalized Chernoff information, which is achieved by optimally designing the detection threshold for the mismatched detector. Furthermore, we have presented a set of equations to compute the optimal detection threshold. We have provided an example of energy detection under the Gauss-Markov signal model. Numerical results show that the maximum error exponent for energy detection ignoring the signal correlation can be close to the original Chernoff information simply by choosing the detection threshold judiciously.

\section{REFERENCES}

[1] Y. Lee and Y. Sung, "The bahadur efficiency for energy detection of stationary gaussian processes," in Proc. ICASSP, Mar. 2012.

[2] D. Kazakos, "Signal detection under mismatch," IEEE Trans. Inf. Theory, vol. 28, pp. 681-684, July 1982.

[3] P. J. Huber, "A robust version of the probability ratio test," Ann. Math. Statist., vol. 36, pp. 1753-1758, Dec. 1965.

[4] S. A. Kassam and H. V. Poor, "Robust techniques for signal processing: A survey," Proc. IEEE, vol. 73, pp. 433-481, Mar. 1985.

[5] E. A. Geraniotis, "Performance bounds for discrimination problem with uncertain statistics," IEEE Trans. Inf. Theory, vol. 31, pp. 703-707, Sept. 1985

[6] J. S. Sadowsky, "Robust large deviations performance analysis for large sample detectors," IEEE Trans. Inf. Theory, vol. 35, pp. 917-920, July 1989.

[7] W. Zhang and H. V. Poor, "On minimax robust detection of stationary Gaussian signals in white Gaussian noise," IEEE Trans. Inf. Theory, vol. 57, pp. 3915-3924, June 2011.

[8] A. Dembo and O. Zeitouni, Large Deviations Techniques and Applications. New York: Springer Verlag, 2009, vol. 38.

[9] Y. Sung, L. Tong, and H. V. Poor, "Neyman-Pearson detection of Gauss-Markov signals in noise: Closed-form error exponent and properties," IEEE Trans. Inf. Theory, vol. 52, pp. 1354-1365, Apr. 2006. 